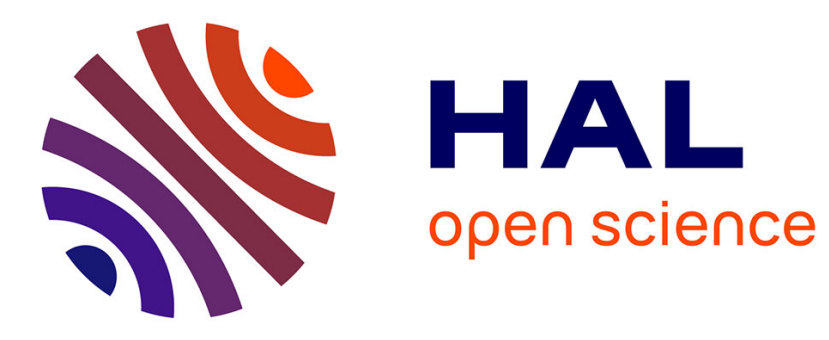

\title{
Offloading Cellular Networks Through V2V Communications - How to Select the Seed-Vehicles?
}

Farouk Mezghani, Riadh Dhaou, Michele Nogueira, André-Luc Beylot

\section{To cite this version:}

Farouk Mezghani, Riadh Dhaou, Michele Nogueira, André-Luc Beylot. Offloading Cellular Networks Through V2V Communications - How to Select the Seed-Vehicles?. IEEE International Conference on Communications (ICC 2016), May 2016, Kuala Lampur, Malaysia. pp.1-6, 10.1109/ICC.2016.7511125 . hal-03155047

\section{HAL Id: hal-03155047 \\ https://hal.science/hal-03155047}

Submitted on 3 Mar 2021

HAL is a multi-disciplinary open access archive for the deposit and dissemination of scientific research documents, whether they are published or not. The documents may come from teaching and research institutions in France or abroad, or from public or private research centers.
L'archive ouverte pluridisciplinaire HAL, est destinée au dépôt et à la diffusion de documents scientifiques de niveau recherche, publiés ou non, émanant des établissements d'enseignement et de recherche français ou étrangers, des laboratoires publics ou privés. 


\title{
Offloading Cellular Networks Through V2V Communications - How to Select the Seed-Vehicles?
}

\author{
Farouk Mezghani*, Riadh Dhaou*, Michele Nogueira ${ }^{\S}$, André-Luc Beylot* \\ *Université de Toulouse, INP/ENSEEIHT, IRIT, France

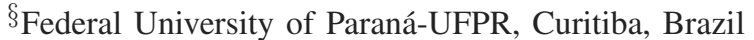 \\ *\{Farouk.Mezghani, Riadh.Dhaou, Andre-Luc.Beylot $\} @$ enseeiht.fr, ${ }^{\S}$ michele@inf.ufpr.br
}

\begin{abstract}
The rapid increase of portable devices providing a multitude of mobile applications have led to excessive cellular traffic demands and consequently to the overload of cellular networks. Recently, migrating this traffic by opportunistic vehicular networks has attracted a great interest and appeared as a promising solution. Indeed, only a limited set of vehicles (seeds) is selected to download objects from an Internet-content server through the cellular network and then propagate the content gradually by opportunistic communications (i.e. vehicleto-vehicle V2V). This paper proposes SIEVE, an innovative seed selection scheme, that exploits two key criteria: users' interests and near-future contacts prediction. Based on these criteria, SIEVE allows to select the seeds in order to maximally satisfy the users' interests and, hence, achieve a maximum content utility (i.e. quantitative metric that determines how satisfied are the users). Simulations results show that SIEVE can improve the content utility when compared to other algorithms.
\end{abstract}

\section{INTRODUCTION}

Due to the proliferation of mobile devices (e.g. smartphones and On-Board Units OBU), vehicular networks have witnessed the development of several applications for commuters (i.e. drivers, passengers) [1-3]. These applications offer a significant convenience for drivers and passengers providing a multitude of information such as touristic recommendation and sale advertisement [4]. Most of these applications enable vehicular users to access and download a large amount of content from an Internet-based content server through cellular networks. One side effect of this tremendous applications spread lies in the inability of cellular networks withstand sudden increases of traffic due to the infrastructure limitations, and it is likely to be overloaded.

Offloading cellular networks through vehicular opportunistic communications (i.e. V2V) has been investigated as a promising solution to partially overcome this problem [5]. Vehicles can use short-range communications, such as DSRC technologies, for sharing and distributing content between them. Hence, initially, only a small set of nodes, called seeds, are selected to download content objects from a content server using the cellular network. A seed carries and broadcasts its objects to its neighboring nodes, and then content can then be gradually propagated all over the network. Hence, this avoids the excessive dependence on cellular networks and benefits the network from V2V communication characterized by its low cost, easy deployment, and higher transmission rate compared to cellular networks.
Selecting seed-vehicles is the initial step for the cellular networks offloading, being also a challenging task [6]. On the one hand, the vehicular network topology is highly dynamic due to the vehicles mobility, making hard to foresee the network topology. On the other hand, a content object may be time-critical (i.e. no longer accurate or relevant after a given period of time), therefore, it should be delivered rapidly to interested users before its lifetime expires.

Several seed selection approaches have been designed for vehicular networks [5], [7-10]. Basically, these works select as seed-vehicles the most influential nodes in the network considering different criteria such node centrality, future contacts prediction, geographic zone, and so on. They only target on delivering the content rapidly to a large number of users with a minimum use of the cellular networks. Although these approaches could achieve good results in terms of delivery ratio and cellular traffic offloading, they may not be able to maximally satisfy the users interests. These approaches do not consider users interests for various content objects that can be generated. Therefore, the goal of this work is to design an interest-aware seed selection scheme for vehicular networks.
(1)

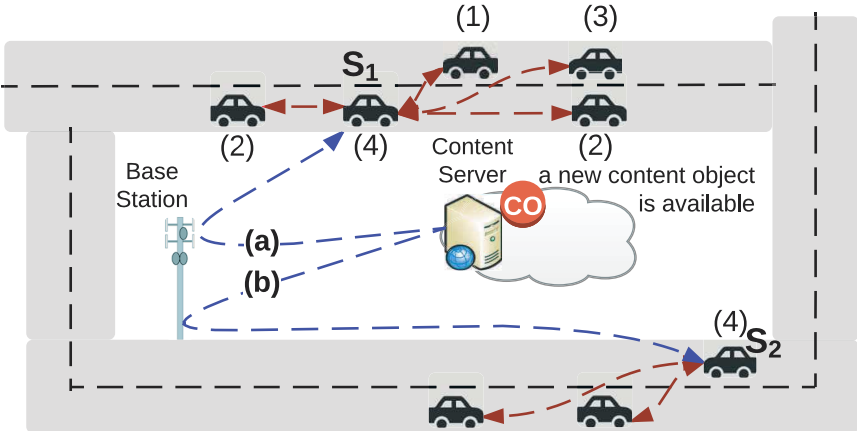

(6)

(6) $\longrightarrow$ Cellular transmission
Consider the example shown in Fig. 1 where a new content object is being created and available within a content server. Each vehicular user has interest (i.e. expressed by integer value as shown in Fig. 1) for the new object. Let us assume, in this scenario, that only one vehicle is assigned as seed. Considering the degree centrality (i.e. the number of other nodes in the graph that the node shares edges with) as the criteria to select 
the most influential nodes, vehicle $S_{1}$ will be chosen as seed since it has the highest number of neighbors. $S_{1}$ will then download the object and diffuse it to its neighboring vehicles and allows then to achieve an utility $U_{S_{1}}=4+2+2+1+3=$ 12 (Fig. 1 (a)). However, such a seed selection strategy cannot achieve the maximal global utility (i.e. utility of the entire network) because it does not consider the users preferences. In particular, considering the vehicle $S_{2}$ as seed (Fig. 1 (b)) can provide more utility even though $S_{2}$ has not the highest degree centrality $\left(U_{S_{2}}=4+6+6=16>12\right)$.

The goal of this paper is thus to develop an interest-aware seed selection approach that maximizes the global utility for all vehicular users. This work presents SIEVE, a seed selection scheme, that considers two important aspects: users preferences and future encounter prediction in order to improve the users satisfaction. SIEVE performance is evaluated through extensive simulations over a 'content utility' metric (i.e. a quantitative metric that computes the users' gain by means of interests in receiving the content). Results demonstrate that SIEVE achieves better content utility against the state-of-theart schemes.

This paper proceeds as follows. Sections II and III present the system model and describe the SIEVE scheme. In Section IV, intensive simulation results demonstrate the effectiveness of SIEVE. Section V reviews the related works. Finally, Section VI presents the conclusion and future works.

\section{System Model}

This work considers a vehicular network composed of mobile users moving on a road topology covered by a cellular infrastructure, as illustrated in Fig. 2. We consider a set of vehicles $\mathcal{V}=\left\{v_{i}\right\}$ equipped with a cellular interface and a short range communication interface (e.g. 802.11p). The system also includes a vehicular controller and an Internetcontent server from which seed-nodes retrieve the content objects, initially through the cellular networks. This work focuses on convenience and commercial applications that offer different services for commuters along vehicular roads. For instance, information from the service providers announcing their services and trying to attract commuters to their stores (e.g. announcements like petrol pumps, highways restaurants, and others). The term content object represents an information regarding a specific service, such as a gas station advertisement. Seeds can then exploit the direct communications (i.e. $\mathrm{V} 2 \mathrm{~V}$ ) to disseminate downloaded content objects to neighboring nodes. Consequently, nodes can store, carry and forward content objects until reaching all interested users.

Each node $v_{i}$ is required to explicitly provide its preferences for different predefined topics $\mathcal{T}=\left\{T_{k}\right\}$ (e.g. gas station offers, restaurant recommendation), being the preferences represented by interest values ranging from 0 to 4 . Indeed, nowadays, users have heterogeneous interests for the different topics (e.g. a user can be interested for both topics $T_{1}$ and $T_{2}$ but s/he may be more interested on $T_{1}$ than for $T_{2}$ ). Let $I_{i, T_{k}} \in[0,4]$ and $I_{i, j} \in[0,4]$ denote the interests of the node $v_{i}$ for the topic $T_{k}$ and the content object $o_{j}$, respectively.
$I_{i, j}=I_{i, T_{k}}$ if $o_{j}$ is a content object regarding the topic $T_{k}$. Hence, when a node $v_{i}$ receives a content object $o_{j}$ regarding a topic $T_{k}, v_{i}$ gets the utility $=I_{i, j}=I_{i, T_{k}}$.

This work considers that a node does not get any utility when getting an object that it had received before (i.e. duplicate object) or if it receives an uninteresting content object (i.e. $I_{i, j}=0$ ).

For the sake of simplicity and without loss of generality, a vehicle is only associated to one user that can be the driver or a passenger. In this paper, the terms 'vehicles' and 'users' are referred to as 'nodes'.

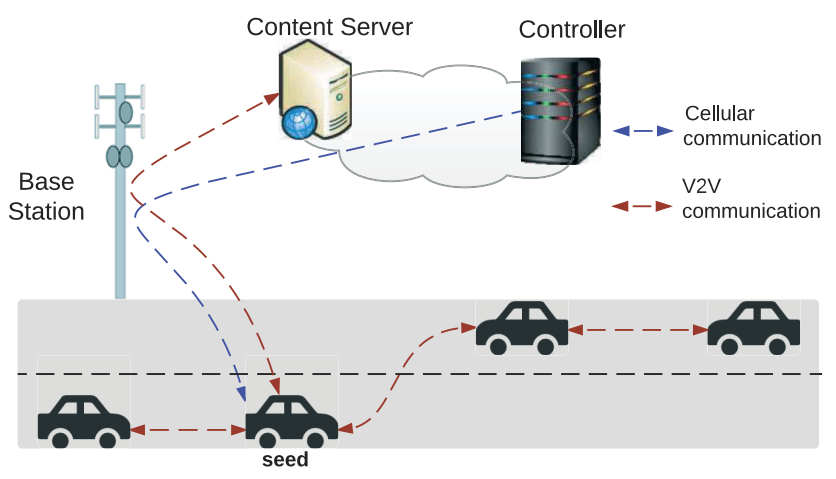

Fig. 2. Network system

Let's assume $o^{*}$ as a new content object created at $T_{e}$, available within the content server and with a lifetime $T_{\max }$. In order to increase the users satisfaction, the efficient seed for $o^{*}$ is the node that can reach a maximum number of interested users and can achieve a maximum utility as shown in eq. 1.

$$
\text { seed }=\underset{v_{k}}{\arg \max } \sum_{v_{i} \text { received } o^{*} \text { before } T_{e}+T_{\max }} I_{i, o^{*}}
$$

It is difficult to resolve this problem unless we know the nodes interests and all the future communications during the object lifetime. To this end, the aforementioned vehicular controller collects periodically information on the position, speed, direction and interests of nodes. By exploiting such information, the vehicular controller predicts the evolution of vehicle movements over a near-future time horizon $\mathrm{H}$, with a time granularity $N \times \delta t(N \in \mathbb{N})$ with a time granularity $\delta t$. The predicated location of vehicles is then exploited by the vehicular controller to determine future V2V contacts over the horizon $N \times \delta t$ [11]. In the following, we stress that the vehicular controller can predict vehicle mobility and contacts.

\section{Discussion: SDN controller an example of vehicular controller}

Recently, some research works [12], [13] showed that the use of the paradigm of Software Defined Networking (SDN) can provide the flexibility and programmability and introduces new services and features to today's vehicular networks. Indeed, vehicles send periodically their information (e.g. speeds, position) to an SDN-controller, allowing it to get a global view of the network, and enabling it to control the network behavior 
by intelligent decisions such as the selection of the efficient seeds in our context.

\section{SIEVE SCHEME}

The main contribution of this paper lies in SIEVE: a Seed selectIon scheme for maximally satisfying commutErs interests in Vehicular nEtworks. Differently from other approaches, SIEVE considers both heterogeneous user interests and future contacts prediction in order to efficiently assign the seednodes. It aims to maximize the content utility.

\section{A. SIEVE phases}

Fig. 3 illustrates the main phases of the SIEVE scheme: (i) vehicles information gathering, (ii) prediction of nodes impacted by $v_{i}$, (iii) content utility computation and seed selection; and ( $i v)$ content objects download. These phases are described as follows.

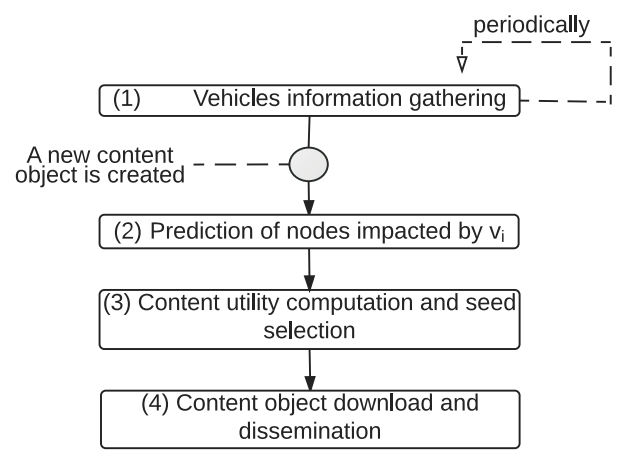

Fig. 3. SIEVE phases

1) Vehicles information gathering: The vehicular controller collects periodically beacon messages from nodes allowing it to get a better view of the network. Beacon messages comprise: $<I D,(x, y), s, \overrightarrow{d i r}, \operatorname{seq}>$

where $I D$ represents the identity of the node; $(x, y)$ indicates the current location of the node; $s$ and $\overrightarrow{d i r}$ are the speed and the direction of the vehicle, respectively; and seq is a sequence of the user interests for the different topics. When a new content object is created, SIEVE starts the next phases of the seed selection process in which the vehicular controller predicts the future nodes that will be impacted by a node $v_{i}$.

2) Prediction of nodes impacted by $v_{i}$ : We consider that the vehicular controller can predict the near-future V2V encounters. Thus it can determine the list of impacted nodes $\mathcal{L}_{v_{i}, T}$ by the $v_{i}$ during a period of time $T$. Impacted nodes refer to the nodes that are likely to receive a content object initially owned by $v_{i}$ before $T$ expires. Fig. 4 illustrates an example of nodes impacted by $v_{1}$ during the period $[t, t+5 \delta t]$ where $\mathcal{L}_{v_{1},[t, t+5 \delta t]}=\left\{v_{i} / i \in[2,10]\right\}$. Thus, if $v_{1}$ owns a content object $o_{j}$ at instant $t$, the nodes $\mathcal{L}_{v_{1},[t, t+5 \delta t]}$ are likely to receive $o_{j}$ in the period of time $[t, t+5 \delta t]$

3) Content utility computation and seed selection: Given a content object $o^{*}$ created at the instant $T_{e}$, in order to maximize the content utility the seed-node of $o^{*}$ is assigned as the node that can produce the maximum utility during the future contact prediction (i.e. $\left[T_{e}, T_{e}+N \times \delta t\right]$ ).

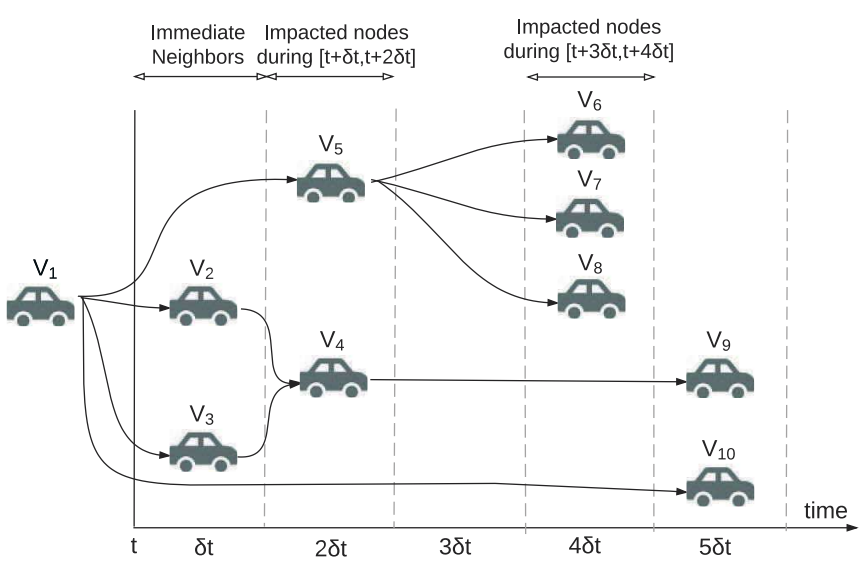

Fig. 4. A sample of contact events of vehicle $v_{1}$ during a period of $5 \delta t$

Knowing the nodes' interests and the list of impacted nodes by $v_{i}$, the vehicular controller computes the content utility that can be produced by $v_{i}$ (Eq. 2).

$$
\operatorname{Utility}\left(v_{i}, o^{*}\right)=\sum_{v_{k} \in \mathcal{L}_{v_{i},\left[T_{e}, T_{e}+N \times \delta t\right]}} I_{k, o^{*}}
$$

Then, the vehicular controller ranks all the nodes, in ascending order according to their content utility values, and chooses as seeds the nodes having the highest content utility values. Finally, the vehicular controller sends a control message for the assigned seeds to perform the final step content object download and dissemination. This study considers that, for the same content object, the number of assigned seeds is greater than or equal to 1 .

4) Content object download and dissemination: When a node, assigned as seed, receives the control message from the vehicular controller, it has to download the content object from the internet-content server through cellular network. Next, this seed disseminates the downloaded content object using the vehicular opportunistic communication (i.e. V2V). Thus, the content object can be gradually disseminated all over the network until its lifetime expires.

This work employs the content utility rate, a quantitative metric that computes the nodes' gain by means of interests (i.e. how satisfied the users are) for the performance evaluation (Eq. 3).

$$
\text { Content Utility rate }=\frac{\sum_{o_{j}} \text { is received by } v_{i} I_{i, j}}{\sum I_{i, j}}
$$

\section{B. SIEVE algorithm}

Algorithm 1 gives the pseudocode for the SIEVE seed selection scheme. It summarizes the SIEVE behavior. This algorithm is executed whenever a new content object is created and available to be disseminated for the different nodes in the network (Line 1). Let $T_{e}$ be the content object creation time. $N$ is set as the number of time slots for future contact prediction (Line 2). 


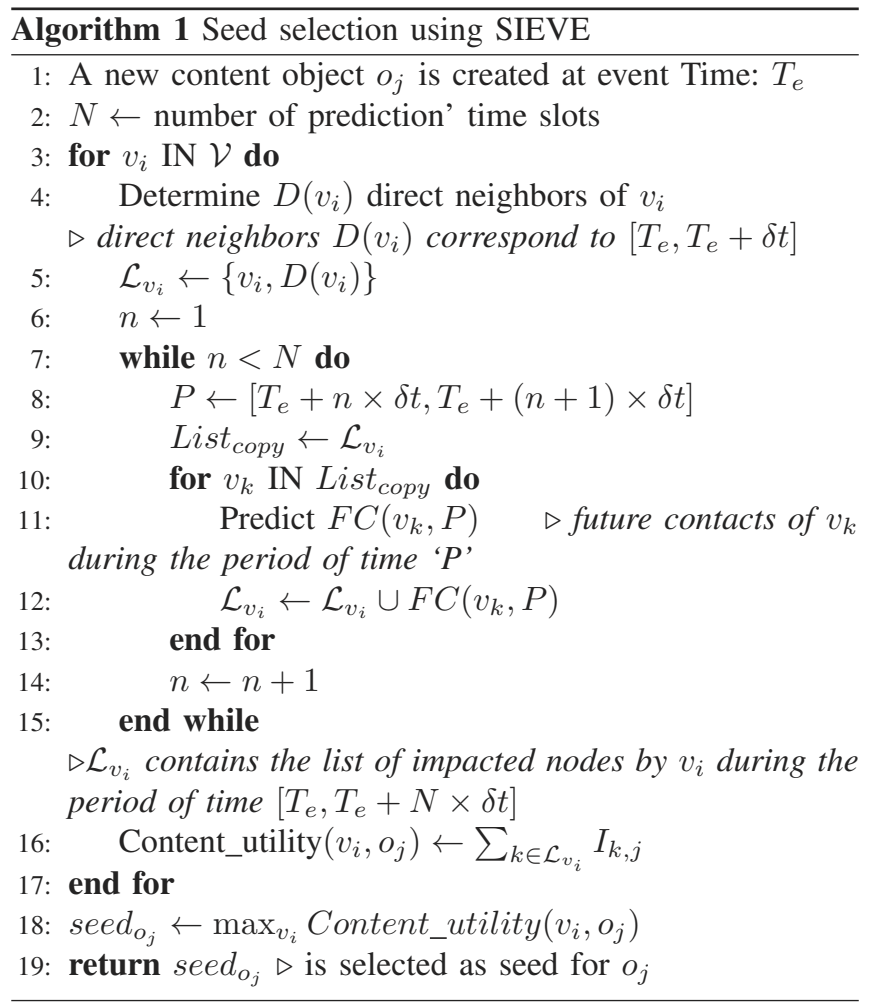

Lines 3-15 allow to determine the list of impacted nodes of each vehicle $v_{i} \in \mathcal{V}$ during $\left[T_{e}, T_{e}+N \delta t\right]$. This is performed analyzing the contact encounters for the different time-slots. Knowing the list of impacted nodes of each vehicle $v_{i}, \mathcal{L}_{v_{i}}$, Line 16 computes the future content utility that $v_{i}$ can produce using $\mathcal{L}_{v_{i}}$ and the nodes interests. Finally, Lines 1819 determine and select the node having the highest content utility value as seed for the content object.

\section{Performance Evaluation}

SIEVE has been evaluated by simulations through the Opportunistic Network Environment (ONE) [14]. Simulations involve a set of vehicles moving with different speeds $[50 \mathrm{~km} / \mathrm{h}, 110 \mathrm{~km} / \mathrm{h}]$ following a vehicular-based map-driven mobility model [15] on a map of Helsinki downtown area of $4500 \mathrm{~m} \times 3400 \mathrm{~m}$ [14]. Each vehicle is equipped with an OBU characterized by a transmission range of $200 \mathrm{~m}$ and a transmission rate of $6 \mathrm{Mbps}$ for the $\mathrm{V} 2 \mathrm{~V}$ communications.

For each user, a list of interests for the different topics is expressed randomly, following a uniform distribution. A number of 100 content objects with equal size $1 \mathrm{MB}$ is generated with an inter-object interval time in a range of $[50,100]$ seconds. Contrarily to the mobile networks where the mobile nodes are equipped with smart devices such as smartphones that have a limited buffer size, vehicular users have OBUs that have large memory size [16]. Therefore, this work assumes that OBUs do not present memory constraints. Furthermore, we consider that OBUs do not have energy constraints since they can be powered by the vehicle's onboard facilities.

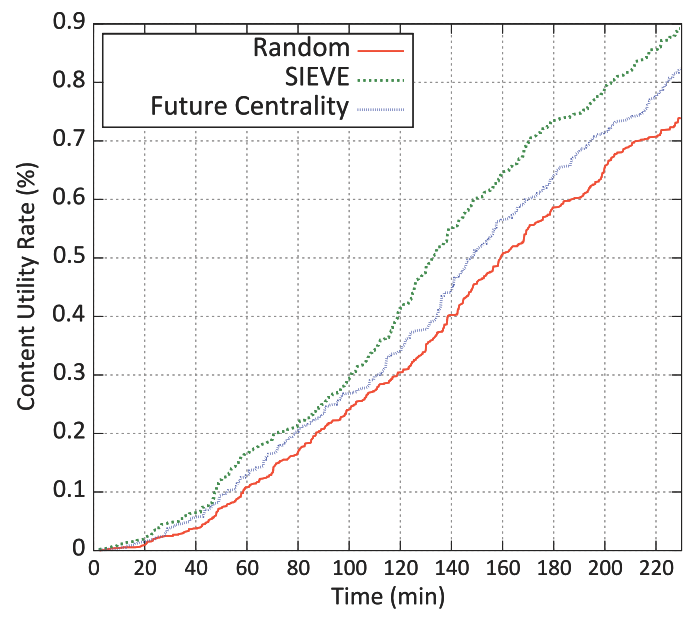

Fig. 5. Cumulative utility over time

The future contacts prediction is performed for a period of 10 time-slots (i.e. $10 \delta t$ ) where $\delta t=4 s$. SIEVE is compared with other seed selection schemes as follows:

- Random: seed-vehicles are chosen randomly. Unlike other methods, the random solution does not use extra information for selecting the seeds.

- Future centrality-based [7]: this approach chooses the seeds as the nodes occupying a vital role in terms of degree centrality, not only in the event time, but also in the future. Authors in [7] have demonstrated that the degree centrality is a good metric to study the dynamics of vehicular sociality as they show both temporal patterns and wide diversity among vehicles.

First simulations are conducted to show the impact of network density on the seed selection schemes. Hence, two scenarios are considered for low and high densities. Scenarios with low density consider a number of 20 vehicles, whereas scenarios with high density consider a number of 100 vehicles. Content objects have equal size of $1 \mathrm{MB}$ and with a lifetime of $60 \mathrm{~min}$, each. For each object, only one node is assigned as seed. Simulations run until all the objects lifetimes expire.

\section{A. Results}

For low density, Fig. 5 shows that SIEVE can achieve a higher content utility of $89 \%$ comparing to future-centrality and random schemes that realize $83 \%$ and $74.5 \%$, respectively. The different approaches could not satisfy all the users' interests before the objects expiration (i.e. the different curves do not converge to $100 \%$ ). In a low density scenario, selecting the seed-vehicles become harder since the vehicles encounter frequency is very low. Thus, as it becomes difficult to diffuse all the content objects before they get expired, then it is important to efficiently choose the seed-vehicles in order to maximally satisfy the users interests.

In a high density scenario, we have noticed that SIEVE produces similar results as future centrality and random methods. The different methods converge to the same content utility rate of $100 \%$ at the same time $(230 \mathrm{~min})$. A high density network 
leads to an increase of the frequency of contacts between the vehicles. Thus, the propagation of a content object can expand rapidly in the core of the network.

Therefore, considering only one seed per object, the seeds selection has no impact on the performance of the content object propagation in a high density network. On the contrary, in low density network, the seeds should be efficiently chosen to maximally satisfy the users interests. Vehicular networks can be considered with low density due to two main reasons: few vehicles are equipped with OBUs (low penetration rate), and the non cooperative behavior of some nodes.

Next, the seed selection schemes performance is evaluated over various TTLs, content objects size, and number of seedvehicles per object for both low and high density scenarios. The results of simulations experiments are shown in Figures 6 and 7. Fig. 6a and Fig. 6b illustrate that SIEVE achieves the best performance followed by Future-centrality and Random considering various TTLs and content object size. Considering various seeds, we check the period of time required, for the different methods, allowing to achieve a content utility rate of $90 \%$ as shown in Fig. 6c SIEVE needs less time to satisfy $90 \%$ from the users interests comparing to Future-centrality and Random. It is thus demonstrated that SIEVE can rapidly propagate the content for the interested users.

Under high density, we have seen that the different methods produce similar results when using 1 seed per object. Thus, we studied if the different methods are similar when limiting the objects lifetime. Fig. 7a shows that, SIEVE outperforms future centrality and random for low TTLs. As the objects lifetime decrease, thus it become harder to receive the content objects before they get expired. Therefore, choosing the seed vehicle that can produce the highest content utility become more important and has more impact on the performance. Fig. 7b and Fig. 7c illustrate that SIEVE overcomes other methods for various content objects size and number of seeds.

Discussion: Vehicular networks appeared with the need of safety applications, and several dissemination protocols are designed for these applications. Nowadays, several research works apply these protocols, designed for safety applications, in the context of comfort applications. However, these two types of applications have different constraints (e.g. comfort applications are less time-constrained compared to safety applications). Therefore, for non-safety applications, there is a need to think about the users convenience and thus to propose other performance metrics to evaluate the users satisfaction.

\section{RELATED WORKS}

Several works [7], [10], [17-20] have investigated the seed selection problem in vehicular networks aiming to improve two main points: (i) maximally offload the cellular traffic and (ii) to expand the content propagation coverage. Some works focused on minimizing the number of seed-vehicles, consequently minimize the cellular communication cost, under the condition that all users can retrieve the content before it expires. Other approaches addressed the choice of the seeds aiming to maximize the coverage of content propagation.
Authors in [21] proposed to choose the most influential nodes (i.e. e those located within the core of the network) in the network in order to ensure an efficient information diffusion. This approach might be efficient in static network but not in very dynamic network as vehicular networks. Thus, to outperform this constraint, the work in [7] proposed to consider the current and the future status of the network. Authors proposed to select the nodes having the highest centrality in the current and future status of the network using the future contact prediction. However, considering different communities, this approach may ignore some communities since it can choose seed-vehicles as nodes with highest centrality but belonging to the same communities. Therefore, a community-based solution [9] have been proposed to select seeds as the nodes having the highest centrality but belonging to different communities.

We do not deny that these works achieved good results in terms of cellular offload percentage and content propagation coverage. However, some of the picture is still missing an important feature: users' interests. In these cited works, a single type of content object is considered and all participating nodes are assumed to be interested in the generated content. Basically, users may be interested in receiving all safety information since it is the commuters life at sake. But for non-safety information, users are seldom interested in the large amount of content that can be generated everyday. They, only want to access to a specific useful content based on their personal preferences [16].

Some works thought to overcome this lack by proposing the geo-dissemination solution in vehicular networks [22] where content are disseminated to destinations confined in specific geographic regions, and thus seed-vehicles can be chosen in certain areas related to the content. Nevertheless, this approach may be only interesting for specific type of content such as traffic information since users are usually interested to receive traffic notification in specific areas. But it is not the case for all type of content (e.g. restaurant recommendation) since they are related to the personal preferences of the user. Therefore, unlike the existing approaches, this work considers heterogeneous users' preferences and aims to maximize the users' satisfaction.

\section{CONCLUSion}

This paper investigates the seed-vehicles selection problem when offloading cellular networks through opportunistic vehicular communications. A novel scheme, called SIEVE, was proposed. Unlike existing works, SIEVE considers heterogeneous users preferences and near-future encounters prediction and aims to maximally satisfy the users interests. The efficiency of SIEVE is emphasized through simulation studies, and results showed that SIEVE outperforms state-of-art schemes. Determining the efficient number of seeds is a focus of our future work. In order to efficiently compute this number, other parameters should be considered such as content popularity (derived from users preferences). 


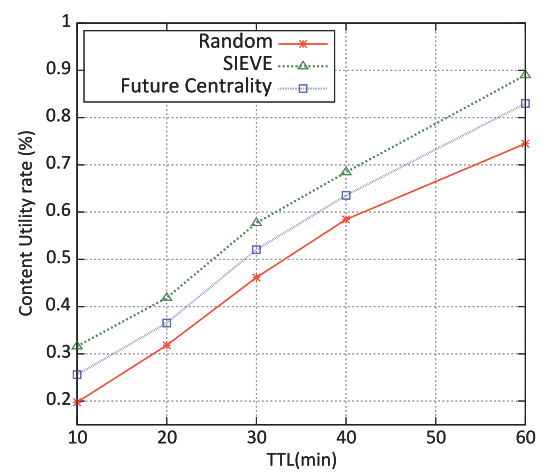

(a) TTL (1 seed; content object size=1MB)

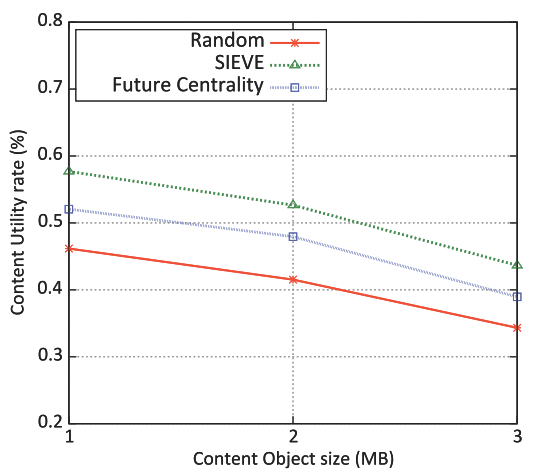

(b) Content object size (TTL=30, 1 seed)

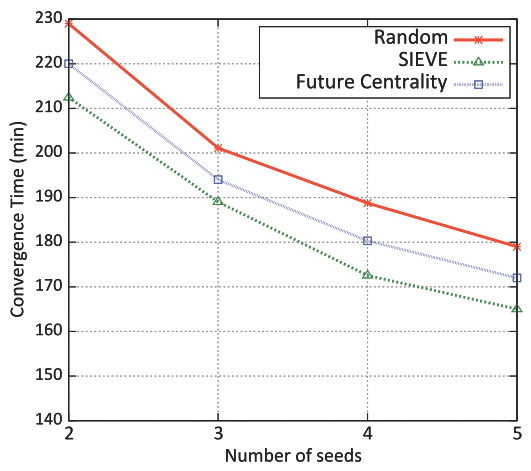

(c) Number of seeds (TTL $=60$, content object size $=1 \mathrm{MB}$, convergence to $90 \%$ of content utility)

Fig. 6. Impact of the TTL, content object size, and number of seeds in a low density scenario

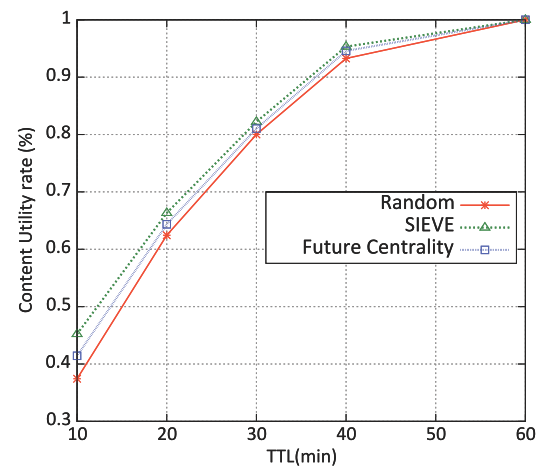

(a) TTL (1 seed; content object size=1MB)

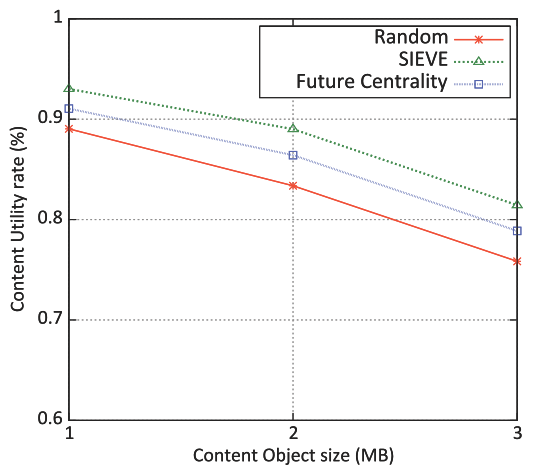

(b) Content object size (TTL $=30,2$ seed)

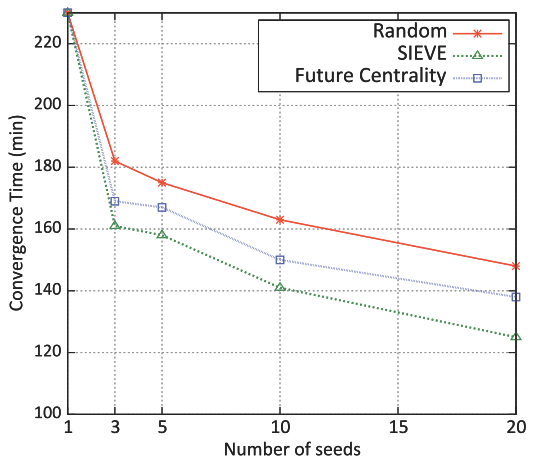

(c) Number of seeds (TTL $=60$, content object size $=1 \mathrm{MB}$, convergence to $100 \%$ of content utility)

Fig. 7. Impact of the TTL, content object size, and number of seeds in a high density scenario

\section{REFERENCES}

[1] "WAZE: Waze Navigation Service." (last access on april 2015). [Online]. Available: https://www.waze.com/

[2] S. Smaldone, L. Han, P. Shankar, and L. Iftode, "RoadSpeak : Enabling Voice Chat on Roadways using Vehicular Social Networks," SocialNets, pp. 43-48, 2008.

[3] W. Sha, D. Kwak, B. Nath, and L. Iftode, "Social Vehicle Navigation: Integrating Shared Driving Experience into Vehicle Navigation," $A C M$ HotMobile, 2013.

[4] H. T. Cheng, H. Shan, and W. Zhuang, "Infotainment and road safety service support in vehicular networking: From a communication perspective," Mech. Syst. Signal Process., Special Issue on Integrated Vehicle Dynamics, vol. 25, no. 6, pp. 2020 - 2038, 2011.

[5] F. Malandrino, C. Casetti, C. Chiasserini, and M. Fiore, "Offloading cellular networks through ITS content download," IEEE SECON, pp. 263-271, 2012.

[6] N. Cheng, N. Lu, N. Zhang, X. Shen, and J. W. Mark, "Vehicular WiFi offloading: Challenges and solutions," Vehicular Communications, vol. 1, no. 1, pp. 13-21, 2014.

[7] J. Qin, H. Zhu, Y. Zhu, L. Lu, G. Xue, and M. Li, "POST: Exploiting Dynamic Sociality for Mobile Advertising in Vehicular Networks," Proc. IEEE INFOCOM, pp. 1761-1769, 2014.

[8] Y. Zhu, Q. Chen, and C. Chen, "Harnessing Vehicle-to-Vehicle Communications for 3G Downloads on the Move," International Journal of Distributed Sensor Networks, vol. 2014, 2014.

[9] Y.-J. Chuang and K. C.-J. Lin, "Cellular traffic offloading through community-based opportunistic dissemination," IEEE WCNC, pp. 3188 3193, 2012.

[10] J. Whitbeck, Y. Lopez, J. Leguay, V. Conan, and M. D. de Amorim, "Push-and-track: Saving infrastructure bandwidth through opportunistic forwarding," Pervasive and Mobile Computing, vol. 8, no. 5, pp. 682$697,2012$.
[11] F. Malandrino, C. Casetti, C. Chiasserini, and M. Fiore, "Content Download in Vehicular Networks in Presence of Noisy Mobility Prediction," IEEE Trans on Mobile Computing, vol. 13, no. 5, pp. 1007-1021, 2014.

[12] I. Ku, Y. Lu, M. Gerla, R. L. Gomes, F. Ongaro, and E. Cerqueira, "Towards software-defined VANET: Architecture and services," MEDHOC-NET, pp. 103-110, 2014.

[13] N. B. Truong, G. M. Lee, and Y. Ghamri-Doudane, "Software defined networking-based vehicular Adhoc Network with Fog Computing," IFIP/IEEE IM, pp. 1202-1207, 2015.

[14] A. Keränen, J. Ott, and T. Kärkkäinen, "The ONE Simulator for DTN Protocol Evaluation," SIMUTools, p. 55, 2009.

[15] A. Keränen, T. Kärkkäinen, and J. Ott, "Simulating Mobility and DTNs with the ONE," Journal of Comm., vol. 5, no. 2, pp. 92-105, 2010.

[16] F. Mezghani, R. Dhaou, M. Nogueira, and A.-L. Beylot, "Content dissemination in Vehicular Social Networks: Taxonomy and User satisfaction," IEEE Comm Magazine, vol. 52, no. 12, pp. 34-40, 2014.

[17] R. Stanica, M. Fiore, and F. Malandrino, "Offloading Floating Car Data," IEEE WoWMoM, pp. 1-9, 2013.

[18] H. Yao, D. Zeng, H. Huang, S. Guo, A. Barnawi, and I. Stojmenovic, "Opportunistic Offloading of Deadline-Constrained Bulk Cellular Traffic in Vehicular DTNs," IEEE Trans on Computers, no. 99, p. 1, 2015.

[19] H. Wang, Y. Zhu, and Q. Zhang, "Compressive sensing based monitoring with vehicular networks," IEEE INFOCOM, pp. 2823-2831, 2013.

[20] R. Kershaw and B. Krishnamachari, "Evaluation of Seed Selection Strategies for Vehicle to Vehicle Epidemic Information Dissemination," IEEE MASS, pp. 581-585, 2014.

[21] M. Kitsak, L. Gallos, S. Havlin, F. Liljeros, L. Muchnik, H. Stanley, and H. Makse, "Identification of influential spreaders in complex networks," Nature Physics, vol. 6, pp. 888-893, 2010.

[22] Y. Li and W. Wang, "Geo-dissemination in vehicular ad hoc networks," IEEE ICC, pp. 302-306, 2012. 